Check for updates

Cite this: RSC Adv., 2021, 11, 3539

Received 22nd December 2020 Accepted 11th January 2021

DOI: 10.1039/dOra10739e

rsc.li/rsc-advances

\section{Visible light-driven Giese reaction with alkyl tosylates catalysed by nucleophilic cobalt $\uparrow$}

\author{
Kimihiro Komeyama, (D) Takuya Michiyuki, Yoshikazu Teshima and Itaru Osaka (D) \\ The scope of the Giese reaction is expanded using readily available alkyl tosylates as substrates and \\ nucleophilic cobalt(I) catalysts under visible-light irradiation. The reaction proceeds preferentially with \\ less bulky primary alkyl tosylates. This unique reactivity enables the regio-selective Giese reaction of \\ polyol derivatives.
}

Organic transformations involving alkyl radicals are powerful tools for the construction of $\mathrm{C}\left(\mathrm{sp}^{3}\right)$-containing carbon-carbon bonds. ${ }^{1}$ Particularly, the conjugate radical addition to electrondeficient olefins (Giese reaction) is a versatile protocol for $\mathrm{C}\left(\mathrm{sp}^{3}\right)-\mathrm{C}\left(\mathrm{sp}^{3}\right)$ bond formation in the synthesis of natural products and pharmaceuticals ${ }^{2}$ because it enables the introduction of alkyl moieties in a site-selective manner.

Typically, the Giese reaction involves the generation of alkyl radicals from organohalides using stannyl radicals, ${ }^{3}$ silyl radicals, ${ }^{4}$ and transition-metal catalysts. ${ }^{5}$ More recently, oxidative protocols have been extensively developed to generate alkyl radicals from alkyl metallic reagents. ${ }^{6}$ However, these approaches are disadvantageous; halogenated waste is generated, and the preparation of most of these alkyl radical sources requires several steps from commercially available chemicals, resulting in a multi-step process to construct the desired carbon-carbon bonds. Therefore, the development of Giese reactions with naturally abundant carbon sources such as alkyl carboxylic acids ${ }^{7}$ and alkyl amines ${ }^{8}$ is receiving increased attention.

Alkanols are also among the most critical carbon sources because they are abundant in bioactive molecules and natural products in the form of sugars, steroids and among others. Furthermore, the hydroxyl substituent plays a vital role as a directing group for $\mathrm{C}-\mathrm{H}$ bond functionalisations. ${ }^{9}$ Therefore, the development of the Giese reaction from alkanols is of great importance in modern synthetic organic chemistry (Scheme 1).

In 2000, Togo reported the first example of a Giese reaction using alkyl xanthates as alkyl radical equivalents (Scheme 1a). ${ }^{\mathbf{1 0}}$ Overman applied a reductive approach for alkyl radical generation from alkyl $N$-phthalimidoyl oxalates by a photocatalyst to the Giese reaction (Scheme 1b). ${ }^{11}$ Similarly, Gong demonstrated

Department of Applied Chemistry, Graduate School of Advanced Science and Engineering, Hiroshima University, 1-4-1 Kagamiyama, Higashi-Hiroshima City, Hiroshima 739-8527, Japan. E-mail: kkome@hiroshima-u.ac.jp

$\dagger$ Electronic supplementary information (ESI) available: Additional experimental results and ${ }^{1} \mathrm{H}$ and ${ }^{13} \mathrm{C}$ NMR spectra data of all products. See DOI: 10.1039/d0ra10739e the reductive strategy of alkyl methyl oxalates as alternative alkyl radical sources according to a unique reaction design. ${ }^{\mathbf{1 2}}$ Moreover, Overman and MacMillan found a radical addition using alkyl cesium oxalates as the source of alkyl radicals (Scheme 1c). ${ }^{13}$ Recently, a direct approach from alkanols to alkyl radicals using a low-valent titanocene catalyst was also demonstrated (Scheme 1d). ${ }^{\mathbf{1 4}}$

These protocols are pioneering works that allowed using abundant alkanols and their derivatives as alkyl radical sources for the Giese reaction. However, since these approaches involve the homolytic cleavage of the robust alcoholic $\mathrm{C}-\mathrm{O}$ bond, the efficiency of the alkyl radical formation greatly depends on the thermodynamic stability of the generated alkyl radicals. Therefore, these Giese reactions are typically limited to tertiary, secondary, and benzylic alkyl radicals as well as heteroatomlinked carbon radicals generated from hemiaminals. Access to non-stabilised primary alkyl radicals from primary alcohols and their derivatives is more challenging.

We recently demonstrated various nickel and nucleophilic cobalt(I)-catalysed transformations of alkyl tosylates, including $\mathrm{C}\left(\mathrm{sp}^{3}\right)-\mathrm{C}\left(\mathrm{sp}^{2}\right)^{15}$ and $\mathrm{C}\left(\mathrm{sp}^{3}\right)-\mathrm{C}\left(\mathrm{sp}^{3}\right)$ coupling $\mathrm{s}^{16}$ and amidation. ${ }^{\mathbf{1 7}}$ These reactions start from an $\mathrm{S}_{\mathrm{N}}$ 2-type oxidative addition of,

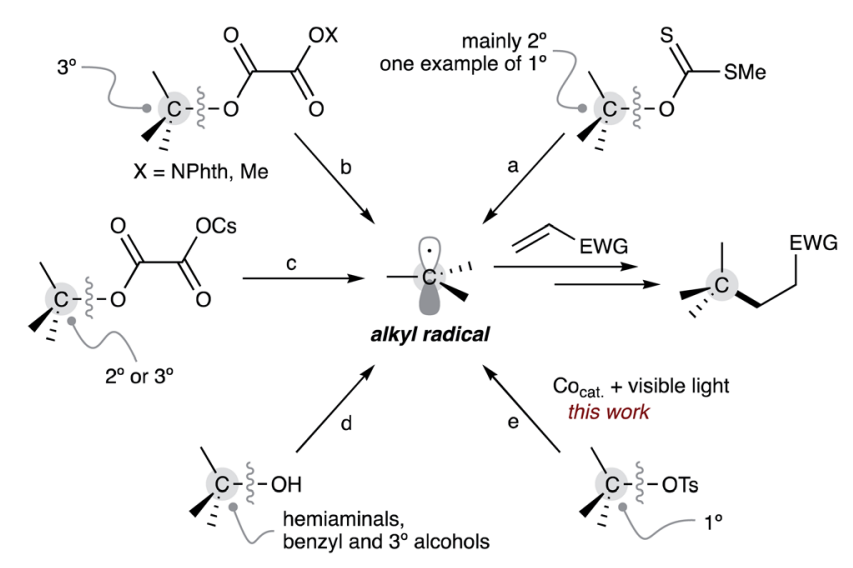

Scheme 1 Examples of alcohol-based Giese reaction. 
especially, primary alkyl tosylates to a nucleophilic cobalt(I) centre, producing an alkyl-cobalt(III) species that enables a transalkylation with a nickel to form a reactive alkyl nickel intermediate. On the other hand, the resulting alkyl-cobalt(III) generates an alkyl radical through homolytic $\mathrm{Co}-\mathrm{C}$ bond cleavage by visible-light irradiation. Numerous radical reactions that involve the catalytic photo-cleavage of a Co-C bond has been developed; ${ }^{18}$ in contrast, the Giese reaction using unactivated alkyl tosylates as alkyl radical sources has never been reported so far. Herein, we report a Giese reaction that uses alkyl tosylates as alkyl radical sources, benefiting from the generation of non-stabilised alkyl radicals by photo-cleavage of the Co-C bond on the corresponding alkyl-cobalt(III) species (Scheme 1e).

Our investigation started by optimising the reaction of alkyl tosylate 1a with activated olefin $\mathbf{2 a}$ (Table 1). When 1a was treated with $2 \mathrm{a}$ (1.0 equiv.) in the presence of vitamin $\mathrm{B}_{12}\left(\mathrm{VB}_{12}\right.$, $5 \mathrm{~mol} \%$ ) as a catalyst, $\mathrm{Mn}$ (3.0 equiv.) as a reductant, and $\mathrm{Et}_{3}$ $\mathrm{N} \cdot \mathrm{HCl}$ (1.5 equiv.) as a proton donor under blue-light irradiation $(\lambda=454 \mathrm{~nm}, 40 \mathrm{~W})$, the expected radical adduct 3a was obtained in $61 \%$ yield (Table 1 , entry 1 ). In the reaction, the presence and type of proton donors greatly affected the reaction efficiency (entries 2-4). The choice of the planar-cobalt catalysts was also crucial. Thus, $\mathrm{CoCl}(\mathrm{dmgH})_{2} \mathrm{Py}, \mathrm{Co}($ salen $)$, and $\mathrm{Co}(\mathrm{Pc})$ catalysts gave the product in negligible yields (entries 5-7),

Table 1 Optimisation of the reaction conditions ${ }^{a}$

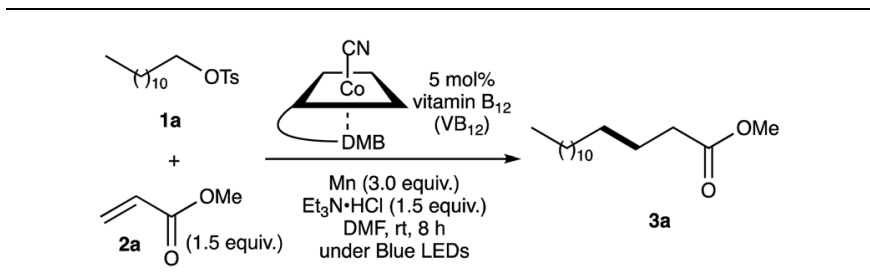

\begin{tabular}{|c|c|c|}
\hline Entry & Changes from standard conditions & Yield $^{b}(\%)$ \\
\hline 1 & None & 61 \\
\hline 2 & Without $\mathrm{Et}_{3} \mathrm{~N} \cdot \mathrm{HCl}$ & 10 \\
\hline 3 & $\mathrm{H}_{2} \mathrm{O}$ instead of $\mathrm{Et}_{3} \mathrm{~N} \cdot \mathrm{HCl}$ & 24 \\
\hline 4 & $\mathrm{NH}_{4} \mathrm{Cl}$ instead of $\mathrm{Et}_{3} \mathrm{~N} \cdot \mathrm{HCl}$ & 54 \\
\hline 5 & $\mathrm{CoCl}(\mathrm{dmgH})_{2} \mathrm{Py}^{c}$ instead of $\mathrm{VB}_{12}$ & Trace \\
\hline 6 & $\mathrm{Co}(\text { salen })^{c}$ instead of $\mathrm{VB}_{12}$ & Trace \\
\hline 7 & $\mathrm{Cp}(\mathrm{Pc})^{c}$ instead of $\mathrm{VB}_{12}$ & Trace \\
\hline 8 & $\mathrm{Co}(\mathrm{TMPP})^{c}$ instead of $\mathrm{VB}_{12}$ & 58 \\
\hline 9 & In ambient conditions ${ }^{d}$ & 15 \\
\hline 10 & In dark & 5 \\
\hline 11 & $\mathrm{Zn}$ instead of $\mathrm{Mn}$ & 51 \\
\hline 12 & Without $\mathrm{VB}_{12}$ & 0 \\
\hline 13 & Without Mn reductant & 0 \\
\hline 14 & Olefin (1.5 equiv.) & $82(80)$ \\
\hline 15 & Olefin (2.0 equiv.) & 70 \\
\hline
\end{tabular}

${ }^{a}$ Standard reaction conditions: $1 \mathrm{a}(0.25 \mathrm{mmol}), \mathbf{2 a}(0.25 \mathrm{mmol}), \mathrm{VB}_{12}$ $(12.5 \mu \mathrm{mol}, 5 \mathrm{~mol} \%), \mathrm{Et}_{3} \mathrm{~N} \cdot \mathrm{HCl}(0.38 \mathrm{mmol})$, Mn powder $(0.75 \mathrm{mmol})$, DMF $(1.5 \mathrm{~mL})$; room temperature for $8 \mathrm{~h}$; the blue-light irradiation; argon atmosphere. ${ }^{b}$ Yields were determined by GC using mesitylene as an internal standard. The parenthesis value indicates the isolated yield. ${ }^{c}$ See ESI. ${ }^{d}$ The reaction was performed without blue-light irradiation. whereas Co(TMPP) afforded the product in 58\% yield (entry 8 ). The present standard conditions gave $3 \mathbf{a}$ in $15 \%$ yield, even under ambient conditions without the light (entry 9). In contrast, the reaction rarely occurred in complete darkness (entry 10). Furthermore, a periodic light-ON/OFF switching experiment in the cobalt-catalysed reaction of 1a with 2a (Fig. 1) and time-dependent UV-Vis absorption spectra of methylcobalamin under blue-light irradiation (Fig. 2) indicated that the photo-induced homolytic cleavage of the $\mathrm{Co}-\mathrm{C}$ bond on the alkyl-Co(III) intermediate produced a Co(II) complex and an alkyl radical. ${ }^{19}$ A slight reduction of the yield was obtained using $\mathrm{Zn}$ instead of Mn (entry 11). Control experiments confirmed that no reaction occurred in the absence of $\mathrm{VB}_{12}$ catalyst (entry 12) and $\mathrm{Mn}$ reductant (entry 13). Finally, increasing the amount of olefin 2a (1.5-2.0 equiv.) improved the yield (70-82\%, entries 14 and 15).

We explored the substrate scope for alkyl tosylates and activated olefins with these optimised reaction conditions in hand (Table 2). Various activated olefins possessing amide, ester, ketone, sulfonyl and pinacolboryl functions were well tolerated in the reaction with some primary alkyl tosylates, producing the target products $\mathbf{3 b} \mathbf{b} \mathbf{3 h}$ in high yields. Notably, the radical addition was performed on a gram-scale to give the desired adduct $\mathbf{3 h}$ in $78 \%$ yield.

Next, the substrate scope for alkyl tosylates was evaluated. Branched alkyl chain (for 3i), aryl ethers (for $\mathbf{3} \mathbf{j}$ and $\mathbf{3 k}$ ), internal (for 31) and terminal alkynyl groups (for $\mathbf{3 m}$ ), a cis-configurated alkenyl group (for 3n) and an ester (for 3o) afforded the corresponding radical adducts in good yields (60-85\%). An alkyl
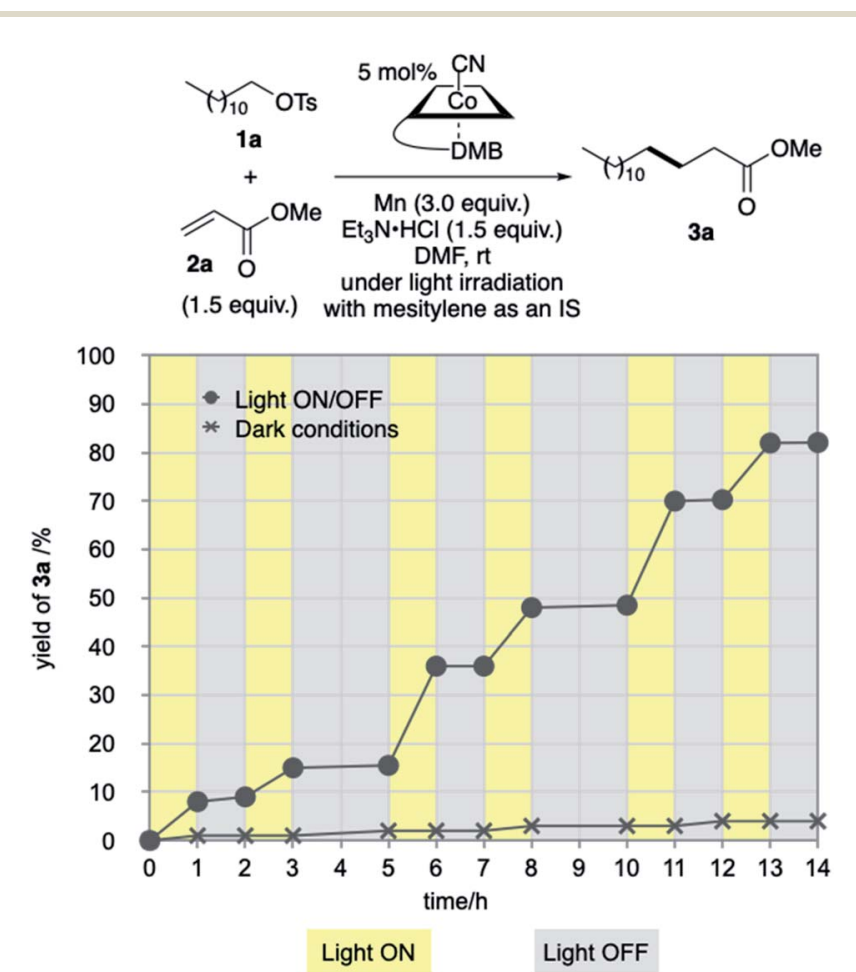

Fig. 1 Plots of product yield (\%) against reaction time (h) in the cobaltcatalysed Giese reaction of $1 \mathrm{a}$ and $2 \mathrm{a}, \mathbf{0}$ : under light ON/OFF conditions, $x$ : in dark. 


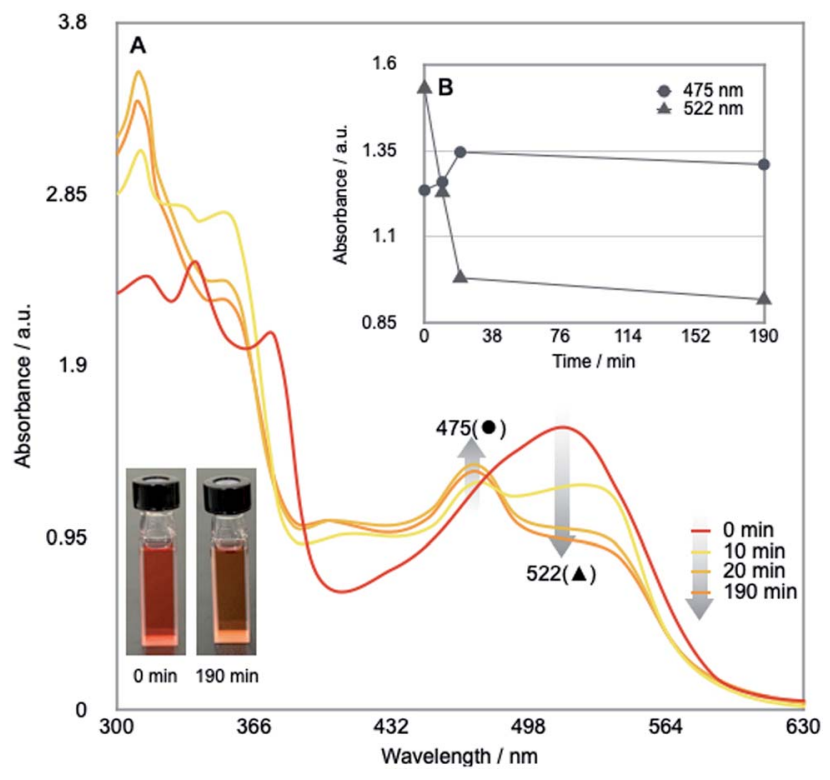

Fig. 2 (A) Time-dependent UV-Vis absorption spectra of Me-Cbl in DMF $\left(10^{-4} \mathrm{~mol} \mathrm{~L}^{-1}\right)$ under the blue-light irradiation under argon atmosphere. (B) Variation over time of absorbances at 475 and $522 \mathrm{~nm}$

chloride was also suitable for this reaction, being converted to radical adduct $\mathbf{3 p}$ in $79 \%$ yield. Interestingly, a chlorosubstituent located at the sterically congested position did not participate in the transformation; chloro-substituted radical adduct 3q was selectively obtained in $75 \%$ yield. In the alkyl radical generation from alkyl tosylates, steric factors can be expected to affect the $\mathrm{S}_{\mathrm{N}} 2$ displacement of nucleophilic cobalt(I). Indeed, the conversion of bulky alkyl tosylates such as 3r-3u was slow under the optimised conditions. The reactivity was improved by increasing the catalyst loading up to $10 \mathrm{~mol} \%$ (for $3 \mathbf{r}$ and 3s) or using a smaller planar-cobalt catalyst like $\mathrm{Co}$ (TMPP) (for $3 \mathbf{t}$ and $3 \mathbf{u}$ ), providing the corresponding adducts in $70-85 \%$ yields, even in the reaction with secondary alkyl tosylate like 3-pyrrolidinyl tosylate. Unfortunately, secondary alkyl tosylates such as cyclohexyl and 2-hexyl tosylates were not compatible with the reaction. The low reactivity associated with the steric repulsion between the cobalt catalyst and the tosylate is a drawback of this reaction; however, it could be also considered a peculiarity. Thus, since in the tosylation of alkyl polyols the tosylation selectively occurs at the primary alcoholic position, ${ }^{20}$ multi-functionalised primary alkyl tosylates could be readily synthesised by the tosylation of polyols and subsequent protection of the remaining hydroxy groups. We believed that a regioselective Giese reaction starting from polyols could be envisaged by combining the present radical addition with the classical tosylation. This strategy enabled the regioselective transformation of various diols bearing primary and secondary alcoholic moieties for the synthesis of functionalised alcohol derivatives $3 \mathbf{v}-\mathbf{3 y}$. Although the present reaction was strongly inhibited by a naked hydroxyl group, pre-treatment of 3-hydroxylbutyl tosylate with trimethylsilyl chloride (1.2 equiv.) in the presence of $\mathrm{Mn}$ (4.2 equiv.) at $25{ }^{\circ} \mathrm{C}$ for $3 \mathrm{~h}$ enabled the direct conversion of the hydroxylated alkyl tosylate to the corresponding radical adduct $3 \mathrm{z}$ in $75 \%$ yield without any deprotection process. A selective Giese reaction of a triol derivative having one TBSO and two TsO groups in different steric environments was also demonstrated, in which the reaction proceeded at the less bulky TsO site without lacking the other TsO group (for 3A).

The reaction of 5-hexenyl tosylate (4) with phenyl acrylate under the standard conditions afforded a mixture of cyclised 5 and linear adducts 6 in 19\% and 34\% yields, respectively (Scheme 2, eqn (1)). Additionally, the $\mathrm{VB}_{12}$-catalysed reaction of dodecyl tosylate (1a) in the presence of $\gamma$-terpinene (3.0 equiv.) as a hydrogen atom donor ${ }^{21}$ afforded dodecane (7) in $80 \%$ GC yield. These results support the generation of alkyl radicals during the reaction. Additionally, in the absence of alkyl tosylate, phenyl acrylate produced diphenyl adipate in $30 \%$ yield and $60 \%$ conversion (Scheme 2, eqn (2)). This result indicates that the nucleophilic cobalt generated in situ could react faster with alkyl tosylates than with activated olefins. ${ }^{22}$

According to these results, we tentatively proposed a catalytic cycle depicted in Scheme 3. The $\mathrm{S}_{\mathrm{N}} 2$-type oxidative addition of alkyl tosylates to nucleophilic cobalt(I) A would afford alkylcobalt(III) B, which would undergo the Co-C bond cleavage under the visible-light irradiation to generate an alkyl radical and planar-cobalt(II) $\mathbf{C}$. The generated alkyl radical would add to the $\beta$-position of an activated olefin, followed by reduction with $\mathrm{Mn}$ and protonation to give adduct 3 . The catalytic cycle would be closed by $\mathrm{Mn}$ reduction of planar-cobalt(II) $\mathbf{C}$ to regenerate the nucleophilic cobalt(I) A.

In conclusion, we have demonstrated a Giese reaction utilising readily available alkyl tosylates as alkyl radical sources for the first time, which proceeded efficiently in the presence of vitamin $\mathrm{B}_{12}$ or $\mathrm{Co}(\mathrm{TTMP})$ catalysts, $\mathrm{Mn}$ reductant, and a proton donor under the visible-light irradiation. The critical step in this reaction is the generation of alkyl-cobalt(III) intermediates by $\mathrm{S}_{\mathrm{N}} 2$ type oxidative addition of alkyl tosylates to nucleophilic cobalt(I). Hence, the bulkiness around the TsO group on alkyl tosylates affected the reaction efficiency. Nevertheless, classical tosylation and subsequent Giese reaction enabled the regioselective functionalisation of polyol compounds. Further studies on the alkyl radical generation protocol to extend the scope of this strategy to novel organic transformations are now ongoing in our laboratory.

\section{Experimental}

\section{General procedure for cobalt-catalysed Giese reaction of alkyl tosylates}

In a Schlenk tube equipped with a stirring bar, Mn powder (41.2 mg, $0.75 \mathrm{mmol}$ ) was added and heated at $400{ }^{\circ} \mathrm{C}$ for $3 \mathrm{~min}$ under vacuum. After cooling and filling with argon, the tube was added triethylamine hydrochloride $\mathrm{Et}_{3} \mathrm{~N} \cdot \mathrm{HCl}(51.6 \mathrm{mg}, 0.38$ $\mathrm{mmol})$ and vitamin $\mathrm{B}_{12}\left(\mathrm{VB}_{12} ; 16.9 \mathrm{mg}, 12.5 \mu \mathrm{mol}\right)$, evacuated and filled with argon three times. After adding dry DMF $(1.5 \mathrm{~mL})$ and stirring for $5 \mathrm{~min}$, the color instantly changed to green by adding trimethylsilyl chloride $(c a .6 \mu \mathrm{L})$. Then, alkyl tosylate $(0.25 \mathrm{mmol})$ and activated olefin $(0.35 \mathrm{mmol})$ were added to the solution. The reaction mixture was stirred at room temperature for the appropriate time (18-24 h) under blue-light irradiation using a PhotoRedOx Box (HeptoChem Inc.) equipped with 
Table 2 Substrate scope of the cobalt-catalysed Giese reaction of alkyl tosylates with activated olefins ${ }^{e}$<smiles>C[14CH2]CCCC(=O)Nc1ccccc1</smiles>

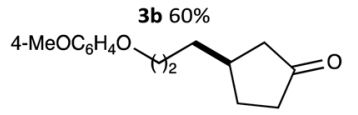

3e $78 \%$<smiles>O=C(CCCNc1ccccc1)Oc1ccccc1</smiles><smiles></smiles><smiles>CCOC(=O)CCCCC(=O)Oc1ccccc1</smiles>

3o $78 \%$<smiles>COC(=O)C(C)CCCC(=O)Oc1ccccc1</smiles><smiles></smiles>

3u $40 \%{ }^{d}(80 \%)^{d, f}$

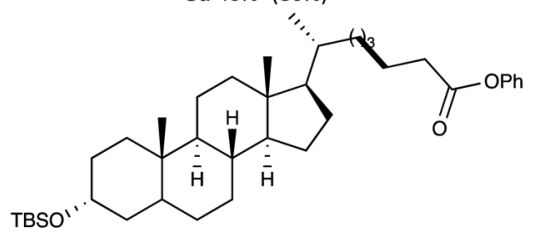

$3 \times 90 \%$<smiles>CCCCCC(=O)N1CCCCC1</smiles>

3c $75 \%$

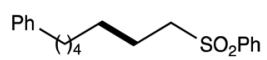

3f $88 \%$<smiles>CC(C)CC(C)CCCCC(=O)Oc1ccccc1</smiles>

$3 \mathbf{i} 77 \%$<smiles>C#CCCCCC(=O)Oc1ccccc1</smiles>

3m $72 \%$<smiles>CCCCCCCC(=O)OCCCCC(=O)Oc1ccccc1</smiles>

3p $82 \%(79 \%)^{c}$<smiles>COC(=O)CCCC([18OH])[18OH]</smiles>
TBSO,<smiles>C[C@H]1CC[C@@H](CCCCC(=O)Oc2ccccc2)C1</smiles>

3v $75 \%$

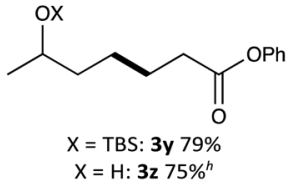<smiles></smiles>

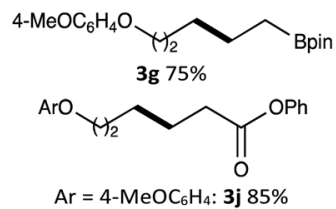

$\mathrm{Ar}=4-\mathrm{ClC}_{6} \mathrm{H}_{4}: 3 \mathbf{k} 79 \%$<smiles>O=C(CCCC/C=C\[18O]c1ccccc1)Oc1ccccc1</smiles><smiles>CC(C)(CCl)C(=O)OCCCCC(=O)Oc1ccccc1</smiles>

3q $75 \%$<smiles>CC1(CCCC(=O)Nc2ccccc2)COC1</smiles>

3t $25 \%^{d}(78 \%)^{d, f}$<smiles>CCCCCCCCCCCCC(=O)Oc1ccccc1</smiles>

3w $75 \%[66: 34]^{9}$<smiles>COC(=O)CCCCC(CO)OC</smiles>

3A $71 \%$

${ }^{a}$ Reaction conditions: alkyl tosylate $(0.25 \mathrm{mmol})$, activated olefin $(0.375 \mathrm{mmol})$, vitamin $\mathrm{B}_{12}(12.5 \mu \mathrm{mol}, 5 \mathrm{~mol} \%), \mathrm{Et} 3 \mathrm{~N} \cdot \mathrm{HCl}(0.38 \mathrm{mmol}), \mathrm{Mn}$ powder $(0.75 \mathrm{mmol})$, DMF $(1.5 \mathrm{~mL})$; room temperature for $16 \mathrm{~h}$; the blue-light irradiation; argon atmosphere. Isolated yield. ${ }^{b}$ The reaction was conducted with 5-phenylpenthyl tosylate $(5 \mathrm{mmol})$ and phenyl acrylate $(7.5 \mathrm{mmol}) .{ }^{c} 5$-(tert-Butyldimethylsilyl)-1-chloropenthane was used instead of the corresponding tosylate. ${ }^{d}$ Reaction time: 24 h. ${ }^{e} 10$ mol\% of vitamin $\mathrm{B}_{12}$ was used. ${ }^{f} \mathrm{Co}(\mathrm{TMPP})$ was used instead of vitamin $\mathrm{B}_{12}$. ${ }^{g}$ Diastereomeric alkyl tosylate $[65: 35]$ was used. The values in bracket indicate a diastereomeric ratio of the radical adduct. ${ }^{h}$ See main text.

a Kessil A160W TUNE BLUE $(\lambda=454 \mathrm{~nm}, 40 \mathrm{~W})$ as shown in Fig. S1 (ESI). $\dagger$ The color of the reaction mixture drastically changed in each step, as depicted in Fig. S2 (ESI). $\dagger$ The obtained mixture was diluted with ethyl acetate and quenched by saturated aqueous $\mathrm{NH}_{4} \mathrm{Cl}$. The aqueous phase was extracted with ethyl acetate, and the combined organic phase was dried over anhydrous $\mathrm{MgSO}_{4}$. After filtration and removal of the solvent, the residue was purified by silica-gel flash column chromatography to give the radical adduct.

\section{Procedure for direct Giese reaction of 3-hydroxylbutyl tosylate: synthesis of $3 z$}

In a Schlenk tube equipped with a stirring bar, Mn powder $(57.7 \mathrm{mg}, 1.05 \mathrm{mmol})$ was added and heated at $400{ }^{\circ} \mathrm{C}$ for $3 \mathrm{~min}$ under vacuum. After cooling and filling with argon, the tube was added $\mathrm{Et}_{3} \mathrm{~N} \cdot \mathrm{HCl}(51.6 \mathrm{mg}, 0.38 \mathrm{mmol})$, DMF $(1.5 \mathrm{~mL})$ and $3-$ hydroxylbutyl tosylate $(61.1 \mathrm{mg}, 0.25 \mathrm{mmol})$. Trimethylsilyl chloride $(38 \mu \mathrm{L}, 0.30 \mathrm{mmol})$ was added into the mixture. The solution was stirred for $3 \mathrm{~h}$ at $25^{\circ} \mathrm{C}$. Then, vitamin $\mathrm{B}_{12}\left(\mathrm{VB}_{12} ; 16.9 \mathrm{mg}, 12.5\right.$ $\mu \mathrm{mol})$ and phenyl acrylate $(55.6 \mathrm{mg}, 0.38 \mathrm{mmol})$ were successively added. The reaction mixture was stirred at room temperature for $16 \mathrm{~h}$ under blue-light irradiation. After reaction, the obtained mixture was diluted with ethyl acetate and quenched by saturated aqueous $\mathrm{NH}_{4} \mathrm{Cl}$. The aqueous phase was extracted with ethyl acetate, and the combined organic phase was dried over anhydrous $\mathrm{MgSO}_{4}$. After filtration and removal of the solvent, the residue was purified by silica-gel flash column chromatography using as $10 \%$ ethyl acetate in hexane an eluent to give the radical adduct $3 z$ ( $41.7 \mathrm{mg}$, colorless oil) in $75 \%$ yield. 


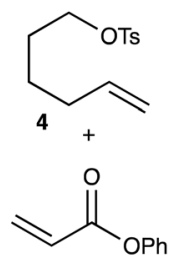

( 1.5 equiv.)
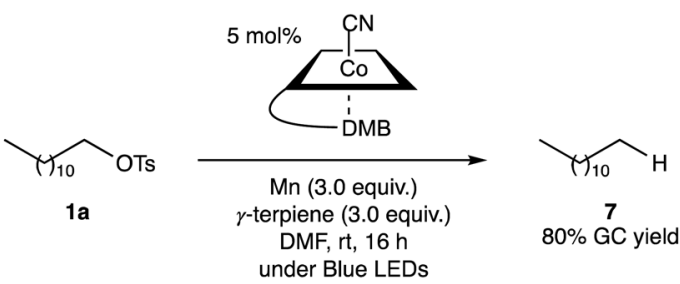

(2)
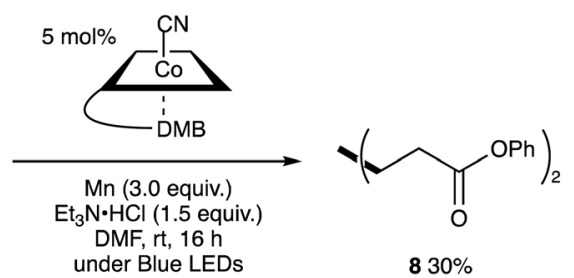

(3)

$60 \%$ conversion

Scheme 2 Radical clock experiment using 5-hexenyl tosylate 6 (eqn (1)). Radical trapping reaction with $\gamma$-terpinene (eqn (2)). Reductive dimerization of phenyl acrylate (eqn (3)).

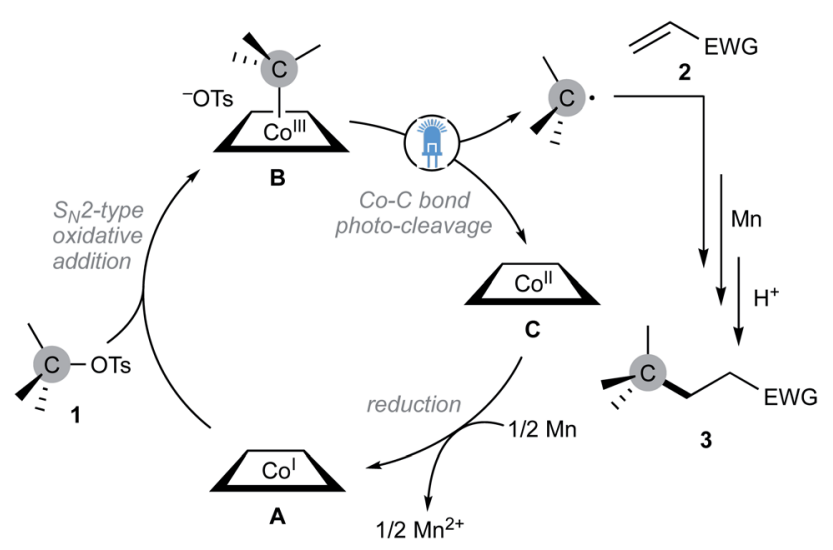

Scheme 3 Plausible reaction mechanism of nucleophilic cobalt-catalysed Giese reaction with alkyl tosylates under the light irradiation.

\section{Characterisation of products}

3a. Isolated as a colorless oil in $80 \%$ yield; ${ }^{1} \mathrm{H}$ NMR $(500$ $\left.\mathrm{MHz}^{\mathrm{CDCl}}{ }_{3}\right) \delta 3.66(\mathrm{~s}, 3 \mathrm{H}), 2.30(\mathrm{t}, J=7.6 \mathrm{~Hz}, 2 \mathrm{H}), 1.66-1.57(\mathrm{~m}$, $2 \mathrm{H}), 1.35-1.16(\mathrm{~m}, 22 \mathrm{H}), 0.87(\mathrm{t}, J=7.0 \mathrm{~Hz}, 3 \mathrm{H}) ;{ }^{13} \mathrm{C}$ NMR $(126$ $\left.\mathrm{MHz}, \mathrm{CDCl}_{3}\right) \delta 174.4,51.4,34.1,31.9,29.7,29.7,29.6,29.6,29.4$, 29.3, 29.2, 29.1, 24.9, 22.7, 14.1; HRMS calcd for $\mathrm{C}_{16} \mathrm{H}_{33} \mathrm{O}_{2}[\mathrm{M}+$ $\mathrm{H}]^{+}:$257.2481, found 257.2479.

3b. Isolated as a colorless oil in $60 \%$ yield; ${ }^{1} \mathrm{H}$ NMR $(500$ $\left.\mathrm{MHz}, \mathrm{CDCl}_{3}\right) \delta 7.51(\mathrm{~d}, J=8.0 \mathrm{~Hz}, 2 \mathrm{H}), 7.35-7.28(\mathrm{~m}, 2 \mathrm{H}), 7.13-$ $7.06(\mathrm{~m}, 1 \mathrm{H}), 2.35(\mathrm{t}, J=7.6 \mathrm{~Hz}, 2 \mathrm{H}), 1.73(\mathrm{p}, J=7.5 \mathrm{~Hz}, 2 \mathrm{H})$,
1.42-1.27 (m, 4H), 1.25 (s, 19H), $0.88(\mathrm{t}, J=6.9 \mathrm{~Hz}, 3 \mathrm{H}) ;{ }^{13} \mathrm{C} \mathrm{NMR}$ $\left(126 \mathrm{MHz}, \mathrm{CDCl}_{3}\right) \delta 171.3,138.0,129.0,124.1,119.7,37.9,31.9$, 29.7, 29.7, 29.6, 29.6, 29.5, 29.4, 29.3, 29.3, 25.6, 22.7, 14.1; HRMS calcd for $\mathrm{C}_{21} \mathrm{H}_{36} \mathrm{NO}[\mathrm{M}+\mathrm{H}]^{+}: 318.2797$, found 304.2642.

3c. Isolated as a colorless oil in $75 \%$ yield; ${ }^{1} \mathrm{H}$ NMR $(500$ $\left.\mathrm{MHz} \mathrm{CDCl}_{3}\right) \delta 3.54(\mathrm{t}, J=5.6 \mathrm{~Hz}, 2 \mathrm{H}), 3.38(\mathrm{t}, J=5.4 \mathrm{~Hz}, 2 \mathrm{H})$, $2.34-2.26(\mathrm{~m}, 2 \mathrm{H}), 1.71-1.44(\mathrm{~m}, 10 \mathrm{H}), 1.24(\mathrm{~s}, 24 \mathrm{H}), 0.87(\mathrm{t}, J=$ $6.8 \mathrm{~Hz}, 3 \mathrm{H}) ;{ }^{13} \mathrm{C} \mathrm{NMR}\left(126 \mathrm{MHz}, \mathrm{CDCl}_{3}\right) \delta 171.5,46.7,42.6,33.5$, 31.9, 29.7, 29.7, 29.6, 29.6, 29.5, 29.5, 29.4, 29.3, 26.6, 25.6, 25.5, 24.6, 22.7, 14.1; HRMS calcd for $\mathrm{C}_{20} \mathrm{H}_{40} \mathrm{NO}[\mathrm{M}+\mathrm{H}]^{+}: 310.3110$, found 310.3108 .

3d. Isolated as a colorless oil in $82 \%$ yield; ${ }^{1} \mathrm{H}$ NMR (500 $\left.\mathrm{MHz} \mathrm{CDCl}_{3}\right) \delta 7.27(\mathrm{t}, J=7.5 \mathrm{~Hz}, 2 \mathrm{H}), 7.17(\mathrm{t}, J=8.8 \mathrm{~Hz}, 3 \mathrm{H})$, $3.69(\mathrm{~s}, 3 \mathrm{H}), 3.67$ (s, 3H), 2.83 (dddd, $J=9.3,7.4,6.3,5.1 \mathrm{~Hz}, 1 \mathrm{H})$, 2.71 (dd, $J=16.5,9.3 \mathrm{~Hz}, 1 \mathrm{H}), 2.62-2.55$ (m, 2H), 2.42 (dd, $J=$ 16.5, $5.2 \mathrm{~Hz}, 1 \mathrm{H}), 1.69-1.56$ (m, 3H), 1.55-1.45 (m, 1H), 1.32 (qt, $J=6.6,2.6 \mathrm{~Hz}, 4 \mathrm{H}) ;{ }^{13} \mathrm{C} \mathrm{NMR}\left(126 \mathrm{MHz}, \mathrm{CDCl}_{3}\right) \delta 175.4,172.4$, 142.5, 128.4, 128.2, 125.6, 51.8, 51.7, 41.1, 35.8, 31.8, 31.2, 29.0, 26.8; HRMS calcd for $\mathrm{C}_{17} \mathrm{H}_{25} \mathrm{O}_{4}[\mathrm{M}+\mathrm{H}]^{+}:$293.1753, found 293.1754 .

3e. Isolated as a colorless oil in $78 \%$ yield; ${ }^{1} \mathrm{H}$ NMR (500 $\left.\mathrm{MHz} \mathrm{CDCl}_{3}\right) \delta 6.83(\mathrm{~s}, 4 \mathrm{H}), 3.92(\mathrm{t}, J=6.3 \mathrm{~Hz}, 2 \mathrm{H}), 3.76(\mathrm{~s}, 3 \mathrm{H})$, 2.46-2.37 (m, 1H), 2.37-2.26 (m, 1H), 2.28-2.10 (m, 3H), 1.89$1.73(\mathrm{~m}, 3 \mathrm{H}), 1.69-1.46(\mathrm{~m}, 3 \mathrm{H}) ;{ }^{13} \mathrm{C} \mathrm{NMR}\left(126 \mathrm{MHz}, \mathrm{CDCl}_{3}\right)$ $\delta$ 219.5, 153.8, 153.1, 115.4, 114.6, 68.4, 55.7, 45.2, 38.5, 37.0, 32.1, 29.5, 27.7; HRMS calcd for $\mathrm{C}_{15} \mathrm{H}_{21} \mathrm{O}_{3}[\mathrm{M}+\mathrm{H}]^{+}$: 249.1491, found 249.1493 .

3f. Isolated as a colorless oil in $88 \%$ yield; ${ }^{1} \mathrm{H}$ NMR $(500 \mathrm{MHz}$, $\left.\mathrm{CDCl}_{3}\right) \delta 7.91(\mathrm{dd}, J=8.4,1.4 \mathrm{~Hz}, 2 \mathrm{H}), 7.69-7.62(\mathrm{~m}, 1 \mathrm{H}), 7.60-$ $7.53(\mathrm{~m}, 2 \mathrm{H}), 7.30-7.23$ (m, 2H), 7.20-7.12 (m, 3H), 3.11-3.04 (m, 2H), 2.61-2.54 (m, 2H), 1.70 (ddt, $J=13.4,10.6,6.4 \mathrm{~Hz}, 2 \mathrm{H})$, 1.61-1.53 (m, 2H), 1.41-1.19 (m, 6H); ${ }^{13} \mathrm{C}$ NMR (126 MHz, $\left.\mathrm{CDCl}_{3}\right) \delta 142.5,139.1,133.6,129.2,128.3,128.2,128.0,125.6$, 56.2, 35.8, 31.2, 28.8, 28.8, 28.1, 22.5; HRMS calcd for $\mathrm{C}_{19} \mathrm{H}_{25} \mathrm{O}_{2} \mathrm{~S}$ $[\mathrm{M}+\mathrm{H}]^{+}: 317.1575$, found 317.1574 .

3g. Isolated as a colorless oil in $75 \%$ yield; ${ }^{1} \mathrm{H}$ NMR $(500$ $\left.\mathrm{MHz}, \mathrm{CDCl}_{3}\right) \delta 6.82(\mathrm{~s}, 4 \mathrm{H}), 3.89(\mathrm{t}, J=6.6 \mathrm{~Hz}, 2 \mathrm{H}), 3.76(\mathrm{~s}, 3 \mathrm{H})$, 1.88 (ddt, $J=16.2,9.6,6.8 \mathrm{~Hz}, 2 \mathrm{H}), 1.75$ (p, $J=6.8 \mathrm{~Hz}, 1 \mathrm{H}), 1.46$ $(\mathrm{dtd}, J=7.8,5.0,2.4 \mathrm{~Hz}, 2 \mathrm{H}), 1.28-1.20(\mathrm{~m}, 13 \mathrm{H}), 0.80(\mathrm{t}, J=$ $7.4 \mathrm{~Hz}, 2 \mathrm{H}) ;{ }^{13} \mathrm{C}$ NMR $\left(126 \mathrm{MHz}, \mathrm{CDCl}_{3}\right) \delta 153.6,153.3,115.4$, 114.6, 82.9, 68.6, 55.7, 29.7, 29.1, 24.8, 23.8, one signal was obscured; HRMS calcd for $\mathrm{C}_{18} \mathrm{H}_{30} \mathrm{BO}_{4}[\mathrm{M}+\mathrm{H}]^{+}: 321.2237$, found 321.2235 .

3h. Isolated as a colorless oil in $81 \%$ yield; ${ }^{1} \mathrm{H}$ NMR (500 $\left.\mathrm{MHz}, \mathrm{CDCl}_{3}\right) \delta$ 7.44-7.35 (m, 2H), 7.35-7.30 (m, 1H), 7.30-7.15 $(\mathrm{m}, 5 \mathrm{H}), 7.14-7.05(\mathrm{~m}, 2 \mathrm{H}), 2.67-2.60(\mathrm{~m}, 2 \mathrm{H}), 2.57(\mathrm{t}, J=7.5 \mathrm{~Hz}$, $2 \mathrm{H}), 1.77(\mathrm{t}, J=7.3 \mathrm{~Hz}, 2 \mathrm{H}), 1.70-1.60(\mathrm{~m}, 2 \mathrm{H}), 1.48-1.34(\mathrm{~m}$, $6 \mathrm{H}) ;{ }^{13} \mathrm{C}$ NMR $\left(126 \mathrm{MHz}, \mathrm{CDCl}_{3}\right) \delta 172.3,150.7,142.7,129.4$, 128.4, 128.2, 125.7, 125.6, 121.5, 35.9, 34.3, 31.4, 29.1, 29.1, 29.0, 24.9; HRMS calcd for $\mathrm{C}_{20} \mathrm{H}_{24} \mathrm{O}_{2}[\mathrm{M}+\mathrm{H}]^{+}:$297.1855, found 297.1850 .

3i. Isolated as a colorless oil in $77 \%$ yield; ${ }^{1} \mathrm{H}$ NMR $(500 \mathrm{MHz}$, $\left.\mathrm{CDCl}_{3}\right) \delta$ 7.41-7.34 (m, 2H), 7.25-7.19 (m, 1H), 7.11-7.05 (m, $2 \mathrm{H}), 2.56(\mathrm{t}, J=7.5 \mathrm{~Hz}, 2 \mathrm{H}), 1.82-1.66(\mathrm{~m}, 2 \mathrm{H}), 1.53(\mathrm{dt}, J=13.3$, $6.6 \mathrm{~Hz}, 1 \mathrm{H}), 1.49-1.03(\mathrm{~m}, 11 \mathrm{H}), 0.87(\mathrm{~d}, J=6.6 \mathrm{~Hz}, 9 \mathrm{H}) ;{ }^{13} \mathrm{C}$ NMR (126 MHz, $\left.\mathrm{CDCl}_{3}\right) \delta 172.3,150.7,129.4,125.7,121.6,39.3$, 
37.2, 36.6, 34.4, 32.6, 28.0, 26.6, 25.3, 24.8, 22.7, 22.6, 19.6; HRMS calcd for $\mathrm{C}_{19} \mathrm{H}_{30} \mathrm{O}_{2}[\mathrm{M}+\mathrm{H}]^{+}:$291.2324, found 291.2326.

3 j. Isolated as a colorless oil in $85 \%$ yield; ${ }^{1} \mathrm{H}$ NMR $(500 \mathrm{MHz}$, $\left.\mathrm{CDCl}_{3}\right) \delta$ 7.42-7.35 (m, 2H), 7.28-7.20 (m, 1H), 7.13-7.05 (m, $2 \mathrm{H}), 6.85(\mathrm{~s}, 3 \mathrm{H}), 3.95(\mathrm{t}, J=6.4 \mathrm{~Hz}, 2 \mathrm{H}), 3.77(\mathrm{~s}, 3 \mathrm{H}), 2.61(\mathrm{t}, J=$ $7.5 \mathrm{~Hz}, 2 \mathrm{H}$ ), 1.84 (ddd, $J=13.7,8.8,6.7 \mathrm{~Hz}, 5 \mathrm{H}$ ), 1.61 (ddt, $J=$ 9.5, 7.0, $2.0 \mathrm{~Hz}, 2 \mathrm{H}) ;{ }^{13} \mathrm{C}$ NMR $\left(126 \mathrm{MHz}, \mathrm{CDCl}_{3}\right) \delta 172.1,153.7$, 153.1, 150.7, 129.4, 125.7, 121.5, 115.4, 114.6, 68.2, 55.7, 34.2, 29.0, 25.6, 24.6; HRMS calcd for $\mathrm{C}_{19} \mathrm{H}_{22} \mathrm{O}_{4}[\mathrm{M}+\mathrm{H}]^{+}$: 315.1596, found 315.1597 .

3k. Isolated as a colorless oil in $79 \%$ yield; ${ }^{1} \mathrm{H}$ NMR $(500$ $\left.\mathrm{MHz} \mathrm{CDCl}_{3}\right) \delta$ 7.42-7.34 (m, 2H), 7.25-7.20 (m, 3H), 7.11-7.05 $(\mathrm{m}, 2 \mathrm{H}), 6.86-6.79(\mathrm{~m}, 2 \mathrm{H}), 3.95(\mathrm{t}, J=6.4 \mathrm{~Hz}, 2 \mathrm{H}), 2.61(\mathrm{t}, J=$ $7.4 \mathrm{~Hz}, 2 \mathrm{H}), 1.89-1.79(\mathrm{~m}, 4 \mathrm{H}), 1.66-1.56(\mathrm{~m}, 2 \mathrm{H}) ;{ }^{13} \mathrm{C}$ NMR $(126$ $\left.\mathrm{MHz}, \mathrm{CDCl}_{3}\right) \delta 172.0,157.6,150.6,129.4,129.2,125.7,125.3$, 121.5, 115.7, 67.8, 34.2, 28.8, 25.5, 24.6.; HRMS calcd for $\mathrm{C}_{18} \mathrm{H}_{20} \mathrm{ClO}_{3}[\mathrm{M}+\mathrm{H}]^{+}$: 319.1101 , found 319.1099.

31. Isolated as a colorless oil in $65 \%$ yield; ${ }^{1} \mathrm{H}$ NMR $(500 \mathrm{MHz}$, $\left.\mathrm{CDCl}_{3}\right) \delta 7.41-7.34(\mathrm{~m}, 2 \mathrm{H}), 7.22(\mathrm{ddt}, J=7.7,7.0,1.1 \mathrm{~Hz}, 1 \mathrm{H})$, 7.11-7.04 (m, 2H), $2.58(\mathrm{dd}, J=7.9,7.1 \mathrm{~Hz}, 2 \mathrm{H}), 2.23(\mathrm{tt}, J=7.0$, $2.4 \mathrm{~Hz}, 2 \mathrm{H}), 2.15(\mathrm{tt}, J=7.1,2.4 \mathrm{~Hz}, 2 \mathrm{H}), 1.91-1.82(\mathrm{~m}, 2 \mathrm{H}), 1.66-$ $1.57(\mathrm{~m}, 2 \mathrm{H}), 1.51-1.34(\mathrm{~m}, 4 \mathrm{H}), 0.90(\mathrm{t}, J=7.2 \mathrm{~Hz}, 3 \mathrm{H}) ;{ }^{13} \mathrm{C} \mathrm{NMR}$ $\left(126 \mathrm{MHz}, \mathrm{CDCl}_{3}\right) \delta 172.0,150.7,129.4,125.7,121.5,80.8,79.3$, 33.9, 31.2, 28.4, 24.1, 21.9, 18.5, 18.4, 13.6; HRMS calcd for $\mathrm{C}_{17} \mathrm{H}_{23} \mathrm{O}_{2}[\mathrm{M}+\mathrm{H}]^{+}:$259.1698, found 259.1696.

3m. Isolated as a colorless oil in $72 \%$ yield; ${ }^{1} \mathrm{H}$ NMR (500 $\left.\mathrm{MHz}, \mathrm{CDCl}_{3}\right) \delta 7.38(\mathrm{t}, J=8.0 \mathrm{~Hz}, 2 \mathrm{H}), 7.22(\mathrm{t}, J=7.4 \mathrm{~Hz}, 1 \mathrm{H})$, 7.07 (d, $J=7.6 \mathrm{~Hz}, 2 \mathrm{H}), 2.58(\mathrm{t}, J=7.5 \mathrm{~Hz}, 2 \mathrm{H}), 2.23(\mathrm{td}, J=6.8$, $2.6 \mathrm{~Hz}, 2 \mathrm{H}), 1.96(\mathrm{t}, J=2.6 \mathrm{~Hz}, 1 \mathrm{H}), 1.78(\mathrm{p}, J=7.4 \mathrm{~Hz}, 2 \mathrm{H}), 1.65-$ $1.49(\mathrm{~m}, 4 \mathrm{H}) ;{ }^{13} \mathrm{C} \mathrm{NMR}\left(126 \mathrm{MHz}, \mathrm{CDCl}_{3}\right) \delta 172.1,150.7,129.4$, 125.7, 121.6, 84.3, 68.4, 34.2, 28.1, 28.1, 24.4, 18.3; HRMS calcd for $\mathrm{C}_{14} \mathrm{H}_{17} \mathrm{O}_{2}[\mathrm{M}+\mathrm{H}]^{+}:$217.1229, found 217.1227.

3n. Isolated as cis-rich stereoisomers (cis/trans $=95: 5$, colorless oil) in $60 \%$ yield; ${ }^{1} \mathrm{H}$ NMR $\left(500 \mathrm{MHz}, \mathrm{CDCl}_{3}\right) \delta 7.41-$ $7.33(\mathrm{~m}, 2 \mathrm{H}), 7.28-7.15(\mathrm{~m}, 1 \mathrm{H}), 7.11-7.04(\mathrm{~m}, 2 \mathrm{H}), 5.40(\mathrm{dtt}, J=$ $10.8,6.8,1.4 \mathrm{~Hz}, 1 \mathrm{H}), 5.36(\mathrm{dtt}, J=10.8,6.9,1.4 \mathrm{~Hz}, 1 \mathrm{H}), 2.57(\mathrm{t}, J$ $=7.4 \mathrm{~Hz}, 2 \mathrm{H}), 2.16-1.99(\mathrm{~m}, 3 \mathrm{H}), 1.83-1.73(\mathrm{~m}, 2 \mathrm{H}), 1.57(\mathrm{~s}, 1 \mathrm{H})$, 1.53-1.43 (m, 2H), 1.38-1.24 (m, 4H), $0.90(\mathrm{t}, J=7.1 \mathrm{~Hz}, 3 \mathrm{H}) ;{ }^{13} \mathrm{C}$ NMR $\left(126 \mathrm{MHz} \mathrm{CDCl}_{3}\right) \delta 172.2,150.7,130.6,129.4,129.0,125.7$, 121.6, 34.3, 31.9, 29.1, 26.9, 26.8, 24.6, 22.3, 14.0; HRMS calcd for $\mathrm{C}_{17} \mathrm{H}_{25} \mathrm{O}_{2}[\mathrm{M}+\mathrm{H}]^{+}$: 261.1855, found 261.1859.

3o. Isolated as a colorless oil in $78 \%$ yield; ${ }^{1} \mathrm{H}$ NMR $(500$ $\left.\mathrm{MHz}, \mathrm{CDCl}_{3}\right) \delta 7.41-7.33(\mathrm{~m}, 2 \mathrm{H}), 7.22(\mathrm{ddt}, J=7.1,6.5,1.1 \mathrm{~Hz}$, $1 \mathrm{H}), 7.10-7.03(\mathrm{~m}, 2 \mathrm{H}), 4.12(\mathrm{q}, J=7.1 \mathrm{~Hz}, 2 \mathrm{H}), 2.55(\mathrm{t}, J=$ $7.5 \mathrm{~Hz}, 2 \mathrm{H}), 2.30(\mathrm{t}, J=7.5 \mathrm{~Hz}, 2 \mathrm{H}), 1.79-1.71(\mathrm{~m}, 2 \mathrm{H}), 1.68-1.57$ (m, 2H), 1.47-1.30 (m, 6H), $1.25(\mathrm{t}, J=7.1 \mathrm{~Hz}, 3 \mathrm{H}) ;{ }^{13} \mathrm{C}$ NMR $(126$ $\left.\mathrm{MHz}, \mathrm{CDCl}_{3}\right) \delta 173.8,172.2,150.7,129.4,125.7,121.5,60.2$, 34.3, 34.3, 28.9, 28.9, 24.9, 24.8, 14.2; HRMS calcd for $\mathrm{C}_{17} \mathrm{H}_{24} \mathrm{O}_{4}$ $[\mathrm{M}+\mathrm{H}]^{+}:$293.1753, found 293.1755.

3p. Isolated as a colorless oil in $82 \%$ yield; ${ }^{1} \mathrm{H}$ NMR (500 $\left.\mathrm{MHz} \mathrm{CDCl}_{3}\right) \delta 7.37(\mathrm{dd}, J=8.5,7.4 \mathrm{~Hz}, 2 \mathrm{H}), 7.27-7.18(\mathrm{~m}, 1 \mathrm{H})$, $7.08(\mathrm{dd}, J=8.7,1.2 \mathrm{~Hz}, 2 \mathrm{H}), 3.61(\mathrm{t}, J=6.6 \mathrm{~Hz}, 2 \mathrm{H}), 2.56(\mathrm{t}, J=$ $7.5 \mathrm{~Hz}, 2 \mathrm{H}), 1.76(\mathrm{p}, J=7.5 \mathrm{~Hz}, 2 \mathrm{H}), 1.58-1.49(\mathrm{~m}, 2 \mathrm{H}), 1.41-1.31$ (m, 6H), $0.90(\mathrm{~s}, 9 \mathrm{H}), 0.06(\mathrm{~s}, 6 \mathrm{H}) ;{ }^{13} \mathrm{C} \mathrm{NMR}\left(126 \mathrm{MHz}, \mathrm{CDCl}_{3}\right)$ $\delta$ 172.3, 150.7, 129.4, 125.7, 121.5, 63.2, 34.4, 32.8, 29.1, 29.0, 26.0, 25.6, 24.9, 18.4, -5.3.; HRMS calcd for $\mathrm{C}_{20} \mathrm{H}_{35} \mathrm{O}_{3} \mathrm{Si}[\mathrm{M}+$ $\mathrm{H}]^{+}$: 351.2355, found 351.2359. 3q. Isolated as a yellow-viscous oil in $75 \%$ yield; ${ }^{1} \mathrm{H}$ NMR (500 $\left.\mathrm{MHz}, \mathrm{CDCl}_{3}\right) \delta 7.41-7.34(\mathrm{~m}, 2 \mathrm{H}), 7.22(\mathrm{td}, J=7.3,1.1 \mathrm{~Hz}, 1 \mathrm{H})$, 7.11-7.04 (m, 2H), $4.15(\mathrm{t}, J=6.5 \mathrm{~Hz}, 2 \mathrm{H}), 3.61(\mathrm{~s}, 2 \mathrm{H}), 2.58(\mathrm{t}, J$ $=7.5 \mathrm{~Hz}, 2 \mathrm{H}), 1.80(\mathrm{p}, J=7.5 \mathrm{~Hz}, 1 \mathrm{H}), 1.72(\mathrm{dq}, J=8.2,6.6 \mathrm{~Hz}$, 0H), 1.56-1.46 (m, 2H), 1.29 (s, 6H); ${ }^{13} \mathrm{C} \mathrm{NMR} \mathrm{(126} \mathrm{MHz,} \mathrm{CDCl}_{3}$ ) $\delta$ 175.0, 172.0, 150.7, 129.4, 125.8, 121.5, 64.7, 52.1, 44.6, 34.2, 28.3, 25.5, 24.5, 23.2; HRMS calcd for $\mathrm{C}_{17} \mathrm{H}_{24} \mathrm{ClO}_{4}[\mathrm{M}+\mathrm{H}]^{+}$: 327.1363 , found 327.1365 .

3r. Isolated as a colorless oil in $85 \%$ yield; ${ }^{1} \mathrm{H}$ NMR $(500 \mathrm{MHz}$, $\left.\mathrm{CDCl}_{3}\right) \delta 7.38(\mathrm{t}, J=7.8 \mathrm{~Hz}, 2 \mathrm{H}), 7.22(\mathrm{t}, J=7.4 \mathrm{~Hz}, 1 \mathrm{H}), 7.08(\mathrm{t}, J$ $=6.6 \mathrm{~Hz}, 2 \mathrm{H}), 3.69(\mathrm{~s}, 3 \mathrm{H}), 2.64(\mathrm{ddt}, J=7.1,4.4,2.3 \mathrm{~Hz}, 1 \mathrm{H})$, 2.60-2.54 (m, 1H), $2.50(\mathrm{p}, J=6.9 \mathrm{~Hz}, 1 \mathrm{H}), 1.93-1.86(\mathrm{~m}, 1 \mathrm{H})$, 1.84-1.68 (m, 2H), 1.61-1.49 (m, 1H), $1.20(\mathrm{~d}, J=7.0 \mathrm{~Hz}, 3 \mathrm{H})$; ${ }^{13} \mathrm{C}$ NMR $\left(126 \mathrm{MHz}, \mathrm{CDCl}_{3}\right) \delta 176.8,171.8,150.6,129.4,125.7$, 121.5, 51.6, 39.2, 34.1, 33.0, 24.3, 22.6, 17.1; HRMS calcd for $\mathrm{C}_{14} \mathrm{H}_{19} \mathrm{O}_{4}[\mathrm{M}+\mathrm{H}]^{+}:$251.1283, found 251.1286.

3s. Isolated as a yellow-viscous oil in $70 \%$ yield; ${ }^{1} \mathrm{H}$ NMR (500 $\left.\mathrm{MHz} \mathrm{CDCl}_{3}\right) \delta 3.67(\mathrm{~s}, 3 \mathrm{H}), 2.28(\mathrm{t}, J=7.6 \mathrm{~Hz}, 2 \mathrm{H}), 1.64-1.54(\mathrm{~m}$, $3 \mathrm{H}), 1.34-1.24(\mathrm{~m}, 12 \mathrm{H}), 1.28-1.19(\mathrm{~m}, 6 \mathrm{H}), 0.88(\mathrm{td}, J=7.0$, $2.3 \mathrm{~Hz}, 6 \mathrm{H}) ;{ }^{13} \mathrm{C} \mathrm{NMR}\left(126 \mathrm{MHz}, \mathrm{CDCl}_{3}\right) \delta 174.3,51.4,37.2,34.5$, 33.5, 33.2, 33.1, 31.9, 29.8, 28.9, 26.6, 23.1, 22.7, 22.2, 14.2, 14.1; HRMS calcd for $\mathrm{C}_{16} \mathrm{H}_{33} \mathrm{O}_{2}[\mathrm{M}+\mathrm{H}]^{+}$: 257.2481, found 257.2492.

3t. Isolated as a red oil in $78 \%$ yield; ${ }^{1} \mathrm{H}$ NMR $(400 \mathrm{MHz}$, $\left.\mathrm{CDCl}_{3}\right) \delta 7.51(\mathrm{~d}, J=7.9 \mathrm{~Hz}, 2 \mathrm{H}), 7.36(\mathrm{~s}, 1 \mathrm{H}), 7.31(\mathrm{t}, J=7.9 \mathrm{~Hz}$, $2 \mathrm{H}), 7.10(\mathrm{t}, J=7.4 \mathrm{~Hz}, 1 \mathrm{H}), 4.43(\mathrm{~d}, J=5.6 \mathrm{~Hz}, 2 \mathrm{H}), 4.35(\mathrm{~d}, J=$ $5.6 \mathrm{~Hz}, 2 \mathrm{H}), 2.41-2.33(\mathrm{~m}, 2 \mathrm{H}), 1.76-1.64(\mathrm{~m}, 4 \mathrm{H}), 1.30(\mathrm{~s}, 3 \mathrm{H})$; ${ }^{13} \mathrm{C}$ NMR (126 MHz, $\left.\mathrm{CDCl}_{3}\right) \delta 170.8,137.8,129.0,124.2,119.8$, 82.7, 39.1, 38.5, 37.7, 23.1, 20.5; HRMS calcd for $\mathrm{C}_{14} \mathrm{H}_{20} \mathrm{NO}_{2}[\mathrm{M}+$ $\mathrm{H}]^{+}:$234.1494, found 234.1491.

3u. Isolated as a colorless oil in $80 \%$ yield; ${ }^{1} \mathrm{H}$ NMR (500 $\left.\mathrm{MHz} \mathrm{CDCl}_{3}\right) \delta 3.60-3.45(\mathrm{~m}, 3 \mathrm{H}), 3.38(\mathrm{dq}, J=10.9,5.0 \mathrm{~Hz}, 3 \mathrm{H})$, 3.24 (dtd, $J=16.5,10.0,7.0 \mathrm{~Hz}, 1 \mathrm{H}), 2.96-2.81$ (m, 1H), 2.41$2.26(\mathrm{~m}, 2 \mathrm{H}), 2.23-2.08(\mathrm{~m}, 1 \mathrm{H}), 2.06-1.93(\mathrm{~m}, 1 \mathrm{H}), 1.84-1.60$ (m, 5H), 1.60-1.49 (m, 4H), $1.45(\mathrm{~s}, 9 \mathrm{H}) ;{ }^{13} \mathrm{C}$ NMR $(126 \mathrm{MHz}$, $\left.\mathrm{CDCl}_{3}\right) \delta 170.6,154.6,79.0,51.5,50.9,46.6,45.7,45.4,42.7,38.8$, $37.9,31.8,31.8,31.0,29.7,28.6,28.5,26.5,25.5,24.5$; HRMS calcd for $\mathrm{C}_{17} \mathrm{H}_{31} \mathrm{~N}_{2} \mathrm{O}_{3}[\mathrm{M}+\mathrm{H}]^{+}$: 311.2335, found 311.2339.

3v. Isolated as a colorless oil in $75 \%$ yield; ${ }^{1} \mathrm{H}$ NMR (500 $\left.\mathrm{MHz}, \mathrm{CDCl}_{3}\right) \delta 7.41-7.35(\mathrm{~m}, 2 \mathrm{H}), 7.22(\mathrm{ddt}, J=7.8,7.0,1.1 \mathrm{~Hz}$, 1H), 7.10-7.05 (m, 2H), $4.20(\mathrm{q}, J=5.9 \mathrm{~Hz}, 1 \mathrm{H}), 2.56(\mathrm{t}, J=$ $7.5 \mathrm{~Hz}, 2 \mathrm{H}), 2.06-1.98(\mathrm{~m}, 1 \mathrm{H}), 1.84-1.65(\mathrm{~m}, 5 \mathrm{H}), 1.62-1.52(\mathrm{~m}$, $1 \mathrm{H}), 1.47-1.31(\mathrm{~m}, 5 \mathrm{H}), 1.17$ (dddd, $J=12.8,8.9,6.2,0.8 \mathrm{~Hz}, 1 \mathrm{H})$, $0.89(\mathrm{~s}, 9 \mathrm{H}), 0.04(\mathrm{~s}, 6 \mathrm{H}) ;{ }^{13} \mathrm{C} \mathrm{NMR}\left(126 \mathrm{MHz}, \mathrm{CDCl}_{3}\right) \delta 172.3$, 150.7, 129.4, 125.7, 121.6, 74.1, 42.6, 37.5, 36.4, 35.3, 34.4, 29.9, 28.0, 25.9, 25.1, 18.1, -4.7, -4.7; HRMS calcd for $\mathrm{C}_{22} \mathrm{H}_{37} \mathrm{O}_{3} \mathrm{Si}[\mathrm{M}$ $+\mathrm{H}]^{+}: 377.2512$, found 77.2510 .

3w. Isolated as a cis/trans-mixture (colorless oil) in $75 \%$ [66 : 34] yield; ${ }^{1} \mathrm{H}$ NMR $\left(500 \mathrm{MHz}, \mathrm{CDCl}_{3}\right) \delta 7.43-7.34(\mathrm{~m}, 2 \mathrm{H})$, $7.22(\mathrm{ddd}, J=8.0,7.0,1.1 \mathrm{~Hz}, 1 \mathrm{H}), 7.08(\mathrm{ddt}, J=7.6,2.0,1.0 \mathrm{~Hz}$, $2 \mathrm{H}), 3.92(\mathrm{q}, J=3.5,3.1 \mathrm{~Hz}, 0.66 \mathrm{H}), 3.56-3.46(\mathrm{~m}, 0.34 \mathrm{H}), 2.56$ (tdd, $J=7.6,3.1,0.9 \mathrm{~Hz}, 2 \mathrm{H}), 1.88-1.81(\mathrm{~m}, 1 \mathrm{H}), 1.79-1.68(\mathrm{~m}$, $3 \mathrm{H}), 1.66-1.56(\mathrm{~m}, 2 \mathrm{H}), 1.40(\mathrm{p}, J=4.0,3.5 \mathrm{~Hz}, 2 \mathrm{H}), 1.34-1.19$ $(\mathrm{m}, 4 \mathrm{H}), 1.22(\mathrm{~s}, 1 \mathrm{H}), 0.90(\mathrm{~d}, J=2.7 \mathrm{~Hz}, 9 \mathrm{H}), 0.06(\mathrm{~s}, 1 \mathrm{H}) ;{ }^{13} \mathrm{C}$ $\operatorname{NMR}\left(126 \mathrm{MHz}, \mathrm{CDCl}_{3}\right) \delta 172.3,172.2,150.8,129.5,129.2,125.8$, 125.5, 121.7, 121.4, 72.0, 71.9, 67.4, 67.2, 36.4, 36.0, 34.6, 34.4, $34.4,34.2,33.3,33.2,31.5,31.3,27.1,26.7,26.5,26.0,26.0,25.9$, 
$25.9,25.9,25.8,25.7,25.2,25.0,18.2,18.1,-4.5,-4.7,-4.7$, -4.9; HRMS calcd for $\mathrm{C}_{23} \mathrm{H}_{39} \mathrm{O}_{3} \mathrm{Si}[\mathrm{M}+\mathrm{H}]^{+}$: 391.2668 , found 391.2668 .

3x. Isolated as a yellow viscous oil in $90 \%$ yield; ${ }^{1} \mathrm{H}$ NMR $(500$ $\left.\mathrm{MHz} \mathrm{CDCl}_{3}\right) \delta 7.37(\mathrm{t}, J=7.7 \mathrm{~Hz}, 2 \mathrm{H}), 7.22(\mathrm{t}, J=7.5 \mathrm{~Hz}, 1 \mathrm{H})$, $7.08(\mathrm{~d}, J=8.6 \mathrm{~Hz}, 2 \mathrm{H}), 3.58(\mathrm{tt}, J=10.5,4.6 \mathrm{~Hz}, 1 \mathrm{H}), 2.55(\mathrm{t}, J=$ $7.5 \mathrm{~Hz}, 2 \mathrm{H}), 1.96(\mathrm{dt}, J=12.4,3.2 \mathrm{~Hz}, 1 \mathrm{H}), 1.88-1.70(\mathrm{~m}, 7 \mathrm{H})$, 1.59-1.51 (m, 2H), 1.50-1.31 (m, 13H), 1.29-0.83 (m, 9H), 0.94$0.86(\mathrm{~m}, 15 \mathrm{H}), 0.64(\mathrm{~s}, 3 \mathrm{H}), 0.06(\mathrm{~s}, 6 \mathrm{H}) ;{ }^{13} \mathrm{C} \mathrm{NMR}(126 \mathrm{MHz}$, $\left.\mathrm{CDCl}_{3}\right) \delta 172.3,150.7,129.3,125.7,121.6,72.8,56.4,56.2$, 42.7, 42.3, 40.2, 40.1, 36.9, 35.8, 35.7, 35.6, 34.6, 34.4, 31.0, 29.6, 28.3, 27.3, 26.4, 26.0, 25.7, 25.0, 24.2, 23.4, 20.8, 18.6, 18.3, 12.0, -4.6; HRMS calcd for $\mathrm{C}_{39} \mathrm{H}_{65} \mathrm{O}_{3} \mathrm{Si}[\mathrm{M}+\mathrm{H}]^{+}: 609.4703$, found 609.4701 .

3y. Isolated as a thin-yellow oil in $79 \%$ yield; ${ }^{1} \mathrm{H}$ NMR $(500$ $\left.\mathrm{MHz} \mathrm{CDCl}_{3}\right) \delta$ 7.41-7.33 (m, 2H), 7.25-7.18 (m, 1H), 7.10-7.03 $(\mathrm{m}, 2 \mathrm{H}), 3.86-3.76(\mathrm{~m}, 1 \mathrm{H}), 2.56(\mathrm{t}, J=7.5 \mathrm{~Hz}, 2 \mathrm{H}), 1.82-1.70(\mathrm{~m}$, $2 \mathrm{H}), 1.56-1.35(\mathrm{~m}, 4 \mathrm{H}), 1.13$ (d, $J=6.1 \mathrm{~Hz}, 3 \mathrm{H}), 0.89$ (s, 9H), 0.05 (s, 3H), 0.05 (s, 3H); ${ }^{13} \mathrm{C}$ NMR (126 MHz, $\left.\mathrm{CDCl}_{3}\right) \delta$ 172.2, 150.7, 129.4, 125.7, 121.6, 68.4, 39.3, 34.4, 25.9, 25.3, 25.0, 23.8, 18.1, -4.4, -4.7; HRMS calcd for $\mathrm{C}_{19} \mathrm{H}_{33} \mathrm{O}_{3} \mathrm{Si}[\mathrm{M}+\mathrm{H}]^{+}$: 337.2199, found 337.2185.

3z. Isolated as a colorless oil in $75 \%$ yield; ${ }^{1} \mathrm{H}$ NMR $(500$ $\left.\mathrm{MHz}, \mathrm{CDCl}_{3}\right) \delta$ 7.41-7.34 (m, 2H), 7.25-7.18 (m, 1H), 7.10-7.05 $(\mathrm{m}, 2 \mathrm{H}), 3.87-3.78(\mathrm{~m}, 1 \mathrm{H}), 2.58(\mathrm{t}, J=7.5 \mathrm{~Hz}, 2 \mathrm{H}), 1.86-1.70(\mathrm{~m}$, 2H), 1.59-1.41 (m, 5H), 1.20 (d, $J=6.1 \mathrm{~Hz}, 3 \mathrm{H}) ;{ }^{13} \mathrm{C}$ NMR $(126$ $\left.\mathrm{MHz}, \mathrm{CDCl}_{3}\right) \delta 172.2,150.7,129.4,125.7,121.5,67.8,38.8,34.3$, 25.2, 24.8, 23.5; HRMS calcd for $\mathrm{C}_{13} \mathrm{H}_{19} \mathrm{O}_{3}[\mathrm{M}+\mathrm{H}]^{+}$: 223.1334, found 223.1339 .

3A. Isolated as a colorless oil in $71 \%$ yield; ${ }^{1} \mathrm{H}$ NMR (500 $\left.\mathrm{MHz} \mathrm{CDCl}_{3}\right) \delta$ 7.81-7.75 (m, 2H), 7.37-7.31 (m, 2H), 3.90-3.77 $(\mathrm{m}, 3 \mathrm{H}), 3.66(\mathrm{~s}, 3 \mathrm{H}), 2.45(\mathrm{~s}, 3 \mathrm{H}), 2.29(\mathrm{t}, J=7.5 \mathrm{~Hz}, 2 \mathrm{H}), 1.64-$ $1.54(\mathrm{~m}, 3 \mathrm{H}), 1.45-1.33(\mathrm{~m}, 1 \mathrm{H}), 1.32-1.18(\mathrm{~m}, 6 \mathrm{H}), 0.83(\mathrm{~s}, 9 \mathrm{H})$, $0.01(\mathrm{~s}, 3 \mathrm{H}),-0.00(\mathrm{~s}, 3 \mathrm{H}) ;{ }^{13} \mathrm{C} \mathrm{NMR}\left(126 \mathrm{MHz} \mathrm{CDCl}_{3}\right) \delta 174.2$, 144.7, 133.0, 129.8, 127.9, 73.1, 69.9, 51.4, 34.0, 33.9, 29.2, 28.9, $25.7,24.8,24.6,21.6,18.0,-4.6,-4.8$; HRMS calcd for $\mathrm{C}_{23} \mathrm{H}_{41} \mathrm{O}_{6} \mathrm{SSi}[\mathrm{M}+\mathrm{H}]^{+}:$473.2393, found 473.2389.

Isolated as a colorless mixture of 5 and $6(5: 6=65: 35)$ in $53 \%$ yield; ${ }^{1} \mathrm{H}$ NMR $\left(500 \mathrm{MHz}, \mathrm{CDCl}_{3}\right) \delta 7.44-7.31(\mathrm{~m}, 2 \mathrm{H}), 7.29-$ $7.18(\mathrm{~m}, 1 \mathrm{H}), 7.14-7.02(\mathrm{~m}, 2 \mathrm{H}), 5.82(\mathrm{ddt}, J=16.9,10.2,6.7 \mathrm{~Hz}$, $0.53 \mathrm{H}), 5.01(\mathrm{dq}, J=17.1,1.7 \mathrm{~Hz}, 0.52 \mathrm{H}), 4.95(\mathrm{ddt}, J=10.2,2.3$, $1.2 \mathrm{~Hz}, 0.53 \mathrm{H}), 2.56(\mathrm{td}, J=7.5,1.0 \mathrm{~Hz}, 2 \mathrm{H}), 2.07$ (tdd, $J=6.7,5.3$, $1.5 \mathrm{~Hz}, 1 \mathrm{H}), 1.88-1.70$ (m, 3H), 1.69-1.48 (m, 2H), 1.48-1.32 (m, $4 \mathrm{H}), 1.18-1.03(\mathrm{~m}, 1 \mathrm{H}) ;{ }^{13} \mathrm{C} \mathrm{NMR}\left(126 \mathrm{MHz}, \mathrm{CDCl}_{3}\right) \delta 172.3,172.2$, 150.7, 150.7, 138.9, 129.4, 125.7, 125.7, 121.6, 121.5, 114.3, 39.8, 35.6, 34.6, 34.3, 33.7, 32.6, 28.9, 28.7, 28.7, 25.1, 24.9, 24.2; HRMS calcd for $\mathrm{C}_{15} \mathrm{H}_{21} \mathrm{O}_{2}[\mathrm{M}+\mathrm{H}]^{+}$: 233.1542, found 233.1538 .

8 (ref. 23). Isolated as a colorless oil in $30 \%$ yield; ${ }^{1} \mathrm{H}$ NMR $\left(500 \mathrm{MHz}, \mathrm{CDCl}_{3}\right) \delta 7.43-7.34(\mathrm{~m}, 4 \mathrm{H}), 7.27-7.20(\mathrm{~m}, 2 \mathrm{H}), 7.12-$ $7.06(\mathrm{~m}, 4 \mathrm{H}), 2.70-2.59(\mathrm{~m}, 4 \mathrm{H}), 1.95-1.84(\mathrm{~m}, 4 \mathrm{H}) ;{ }^{13} \mathrm{C}$ NMR $\left(126 \mathrm{MHz}, \mathrm{CDCl}_{3}\right) \delta 171.7,150.6,129.4,125.8,121.5,34.0,24.3$.

\section{Periodic light ON/OFF switching experiment in the cobalt- catalysed Giese reaction (Fig. 1)}

In a Schlenk tube equipped with a stirring bar, Mn powder (41.2 $\mathrm{mg}, 0.75 \mathrm{mmol}$ ) was added and heated at $400{ }^{\circ} \mathrm{C}$ for $3 \mathrm{~min}$ under vacuum. After cooling and filling with argon, the tube was charged with $\mathrm{Et}_{3} \mathrm{~N} \cdot \mathrm{HCl}(51.6 \mathrm{mg}, 0.38 \mathrm{mmol})$ and $\mathrm{VB}_{12}(16.9 \mathrm{mg}$, $12.5 \mu \mathrm{mol})$, evacuated and filled with argon for three times. After the addition of dry DMF $(1.5 \mathrm{~mL})$, trimethylsilyl chloride (ca. $6 \mu \mathrm{L})$, and mesitylene $(12.0 \mathrm{mg}, 0.15 \mathrm{mmol})$ as an internal standard (IS), the reaction mixture was stirred for $10 \mathrm{~min}$. The tube was attached to an argon-filled balloon and moved to a light-tight room. Then, dodecyl tosylate (1a, $85.1 \mathrm{mg}, 0.25$ $\mathrm{mmol}$ ) and methyl acrylate (2a, $32.3 \mathrm{mg}, 0.38 \mathrm{mmol})$ were added into the solution. The light ON/OFF experiment was conducted under a periodic photo-irradiation with the photo-reaction apparatus mentioned above at the ambient temperature for $14 \mathrm{~h}$. The product yield was determined by GC measurement of an aliquot of the reaction mixture in each reaction time. The Giese reaction under dark conditions was carried out in the same procedure.

\section{Time-dependent UV-Vis absorption spectra of Me-Cbl under the blue-light irradiation (Fig. 2)}

The UV-Vis absorption spectra methylcobalamin (Me-Cbl) in DMF $\left(10^{-4} \mathrm{~mol} \mathrm{~L}^{-1}\right)$ were recorded on the Shimadzu UV-3600 spectrometer under argon atmosphere in a light-tight room, as depicted in Fig. S4. $\dagger$ Usually, cobalamin(III) and (II) species exhibit red and orange colors in solution, respectively. ${ }^{6}$ Before the blue-light irradiation ( $0 \mathrm{~min}$ ), the solution showed red color and a strong absorption peak at $522 \mathrm{~nm}$ (red line). As the solution was exposed to the blue-light, the above-mentioned peak decreased and a new peak at $475 \mathrm{~nm}$ appeared. After the blue-light irradiation for $190 \mathrm{~min}$, the color of the solution turned to orange. The results would indicate that the blue-light irradiation of Me-Cbl affords a Co(II) complex with methyl radical through the cobalt-carbon bond cleavage.

\section{Radical trapping reaction with $\gamma$-terpinene (eqn (2) in Scheme 2)}

In a Schlenk tube equipped with a stirring bar, Mn powder (41.2 mg, $0.75 \mathrm{mmol}$ ) was added and heated at $400{ }^{\circ} \mathrm{C}$ for $3 \mathrm{~min}$ under vacuum. After cooling and filling with argon, the tube was added vitamin $\mathrm{B}_{12}\left(\mathrm{VB}_{12} ; 16.9 \mathrm{mg}, 12.5 \mu \mathrm{mol}\right)$, evacuated and filled with argon three times. After adding dry DMF $(1.5 \mathrm{~mL})$ and trimethylsilyl chloride (ca. $6 \mu \mathrm{L}$ ), the mixture was stirred for $5 \mathrm{~min}$. Then, dodecyl tosylate (1a, $85.1 \mathrm{mg}, 0.25 \mathrm{mmol}$ ) was added to the solution. The reaction mixture was stirred at room temperature for $16 \mathrm{~h}$ under blue-light irradiation. The obtained mixture was diluted with ethyl acetate and quenched by saturated aqueous $\mathrm{NH}_{4} \mathrm{Cl}$. The product 7 was characterised by an authentic sample. The yield was evaluated by GC measurement using mesitylene as an internal standard.

\section{Conflicts of interest}

There are no conflicts to declare.

\section{Acknowledgements}

This work was supported by JSPS KAKENHI (grant number: JP15K05106). 


\section{Notes and references}

1 (a) C. K. Prier, D. A. Rankic and D. W. C. MacMillan, Chem. Rev., 2013, 113, 5322-5363; (b) M. Yan, J. C. Lo, J. T. Edwards and P. S. Baran, J. Am. Chem. Soc., 2016, 138, 12692-12714; (c) J. Choi and G. C. Fu, Science, 2017, 356, 152-160.

2 (a) G. S. C. Srikanth and S. L. Castle, Tetrahedron, 2005, 61, 10377-10441; (b) J. Streuff and A. Gansäuer, Angew. Chem., Int. Ed., 2015, 54, 14232-14242; (c) G. J. Lovinger and J. P. Morken, Eur. J. Org. Chem., 2020, 2362-2368.

3 B. Giese, J. A. González-Gómez and T. Witzel, Angew. Chem., Int. Ed., 1984, 23, 69-70.

4 M. Ballestri, C. Chatgilialoglu, K. B. Clark, D. Griller, B. Giese and B. Kopping, J. Org. Chem., 1991, 56, 678-683.

5 (a) R. Scheffold, S. Abrecht, R. Orlinski, H. R. Ruf, P. Stamouli, O. Tinembart, L. Walder and C. Weymuth, Pure Appl. Chem., 1987, 59, 363-372; (b) H. Gong, R. S. Andrews, J. L. Zuccarello, S. J. Lee and M. R. Gagné, Org. Lett., 2009, 11, 879-882; (c) X. Wu, W. Hao, K.-Y. Ye, B. Jiang, G. Pombar, Z. Song and S. Lin, J. Am. Chem. Soc., 2018, 140, 14836-14843.

6 (a) G. Sorin, R. Martinez Mallorquin, Y. Contie, A. Baralle, M. Malacria, J. Goddard and L. Fensterbank, Angew. Chem., Int. Ed., 2010, 49, 8721-8723; (b) V. Corcé, L. Chamoreau, E. Derat, J. Goddard, C. Ollivier and L. Fensterbank, Angew. Chem., Int. Ed., 2015, 54, 11414-11418.

7 (a) L. Chu, C. Ohta, Z. Zuo and D. W. C. MacMillan, J. Am. Chem. Soc., 2014, 136, 10886-10889; (b) T. Qin, L. R. Malins, J. T. Edwards, R. R. Merchant, A. J. E. Novak, J. Z. Zhong, R. B. Mills, M. Yan, C. Yuan, M. D. Eastgate and P. S. Baran, Angew. Chem., Int. Ed., 2017, 56, 260-265; (c) N. P. Ramirez and J. C. Gonzalez-Gomez, Eur. J. Org. Chem., 2017, 2154-2163.

8 J. Wu, P. S. Grant, X. Li, A. Noble and V. K. Aggarwal, Angew. Chem., Int. Ed., 2019, 58, 5697-5701.

9 (a) A. Hu, J.-J. Guo, H. Pan, H. Tang, Z. Gao and Z. Zuo, J. Am. Chem. Soc., 2018, 140, 1612-1616; (b) I. Kim, B. Park, G. Kang, J. Kim, H. Jung, H. Lee, M. Baik and S. Hong, Angew. Chem., Int. Ed., 2018, 57, 15517-15522; (c) Y. Zhu, K. Huang, J. Pan, X. Qiu, X. Luo, Q. Qin, J. Wei, X. Wen, L. Zhang and N. Jiao, Nat. Commun., 2018, 9, 2625; (d) C. Karmel, B. Li and J. F. Hartwig, J. Am. Chem. Soc., 2018, 140, 1460-1470.

10 H. Togo, S. Matsubayashi, O. Yamazaki and M. Yokoyama, J. Org. Chem., 2000, 65, 2816-2819.
11 G. L. Lackner, K. W. Quasdorf and L. E. Overman, J. Am. Chem. Soc., 2013, 135, 15342-15345.

12 Y. Ye, H. Chen, J. L. Sessler and H. Gong, J. Am. Chem. Soc., 2019, 141, 820-824.

13 C. C. Nawrat, C. R. Jamison, Y. Slutskyy, D. W. C. MacMillan and L. E. Overman, J. Am. Chem. Soc., 2015, 137, 1127011273.

14 (a) X. Zheng, X. J. Dai, H. Q. Yuan, C. X. Ye, J. Ma and P. Q. Huang, Angew. Chem., Int. Ed., 2013, 52, 3494-3498; (b) T. Suga, S. Shimazu and Y. Ukaji, Org. Lett., 2018, 20, 5389-5392; (c) H. Xie, J. Guo, Y.-Q. Wang, K. Wang, P. Guo, P.-F. Su, X. Wang and X.-Z. Shu, J. Am. Chem. Soc., 2020, 142, 16787-16794.

15 (a) K. Komeyama, R. Ohata, S. Kiguchi and I. Osaka, Chem. Commun., 2017, 53, 6401-6404; (b) K. Komeyama, Y. Yamahata and I. Osaka, Org. Lett., 2018, 20, 4375-4378.

16 (a) K. Komeyama, T. Michiyuki and I. Osaka, ACS Catal., 2019, 9, 9285-9291; (b) K. Komeyama, R. Tsunemitsu, T. Michiyuki, H. Yoshida and I. Osaka, Molecules, 2019, 24, 1458.

17 T. Michiyuki, I. Osaka and K. Komeyama, Chem. Commun., 2020, 56, 1247-1250.

18 (a) M. E. Weiss, L. M. Kreis, A. Lauber and E. M. Carreira, Angew. Chem., Int. Ed., 2011, 50, 11125-11128; (b) G. P. Cerai and B. Morandi, Chem. Commun., 2016, 52, 9769-9772; (c) M. Ociepa, O. Baka, J. Narodowiec and D. Gryko, Adv. Synth. Catal., 2017, 359, 3560-3565; (d) M. J. Hossain, T. Ono, K. Wakiya and Y. Hisaeda, Chem. Commun., 2017, 53, 10878-10881; (e) L. Chen, Y. Kametani, K. Imamura, T. Abe, Y. Shiota, K. Yoshizawa, Y. Hisaeda and H. Shimakoshi, Chem. Commun., 2019, 55, 1307013073; $(f)$ L. Chen, Y. Hisaeda and H. Shimakoshi, Adv. Synth. Catal., 2019, 361, 2877-2884; $(g)$ M. J. Hossain, T. Ono, Y. Yano and Y. Hisaeda, ChemElectroChem, 2019, 6, 4199-4203; (h) M. Ociepa, A. J. Wierzba, J. Turkowska and D. Gryko, J. Am. Chem. Soc., 2020, 142, 5355-5361.

19 S. Izumi, H. Shimakoshi, M. Abe and Y. Hisaeda, Dalton Trans., 2010, 39, 3302-3307.

20 T. W. Greene and P. G. M. Wuts, Protective Groups in Organic Synthesis, Wiley, New York, 3rd edn, 1999.

21 K. C. MacLeod, B. O. Patrick and K. M. Smith, Organometallics, 2010, 29, 6639-6641.

22 G. N. Schrauzer and R. J. Windgassen, J. Am. Chem. Soc., 1967, 89, 1999-2007.

23 Y. Shi, P. C. J. Kamer, D. J. Cole-Hamilton, M. Harvie, E. F. Baxter, K. J. C. Lim and P. Pogorzelec, Chem. Sci., 2017, 8, 6911-6917. 